\title{
Analytical framework for optimized feature extraction for upgrading occupancy sensing performance
}

\author{
Preethi K. Mane, K. Narasimha Rao \\ Department of Electronics and Instrumentation Engineering, BMS College of Engineering, India
}

\begin{tabular}{l} 
Article Info \\
\hline Article history: \\
Received Oct 9, 2019 \\
Revised Nov 25, 2019 \\
Accepted Dec 1, 2019 \\
\hline
\end{tabular}

\section{Keywords:}

Doppler radar

Machine learning

Motion sensing

Occupancy sensing

Optimization

Surveillance

\begin{abstract}
The adoption of the occupancy sensors has become an inevitable in commercial and non-commercial security devices, owing to their proficiency in the energy management. It has been found that the usages of conventional sensors is shrouded with operational problems, hence the use of the Doppler radar offers better mitigation of such problems. However, the usage of Doppler radar towards occupancy sensing in existing system is found to be very much in infancy stage. Moreover, the performance of monitoring using Doppler radar is yet to be improved more. Therefore, this paper introduces a simplified framework for enriching the event sensing performance by efficient selection of minimal robust attributes using Doppler radar. Adoption of analytical methodology has been carried out to find that different machine learning approaches could be further used for improving the accuracy performance for the feature that has been extracted in the proposed system of occuancy system.
\end{abstract}

Copyright (C) 2020Institute of Advanced Engineering and Science. All rights reserved.

\section{Corresponding Author:}

Preethi K. Mane,

Department of Electronics and Instrumentation Engineering,

BMS College of Engineering,

Bangalore, India.

E-Mail: pkmph2008@gmail.com

\section{INTRODUCTION}

The implementation of the various occupancy sensors is widely found in commercial and non-commercial applications [1]. These sensors are of low cost and effective in identifying object based disturances in the surveillance area [2]. At present, occupancy sensing operation is carried out by various sensors e.g. passive infrared sensor, ultrasonic sensor (active and passive), microwave-based identification, etc [3]. Usage of such conventional sensors is reported to offer issues associated with the accuracy of the sensed information [4]. The problem with such sensors is that they offer maximized occurrences of outliers. As a result, the propoer execution of the occupancy sensor to ensure reliable operation becomes a challenging task. In this case, the adoption of radar based mechanism can be expected to offer better solution which works on equivalent principles of reflection of sound. Any radar system makes use of electromagnetic waves, which are transmitted and then studied based on their behaviour of orientation with respect to speed, distance, and direction. The transmitted signal travels through air at the speed of light and it always travels in straight lines until and unless it encounters impediments like a surface that is electrically disruptive. The system can compute the distance which basically represents time delay for the two oppositely scattered signals. Different researches have indicated the significant implementation of Doppler radar to perform the identification of human motion related biosignals [5,6]. The proper usage of these bio-signal helps to know the precise detection of the human body motion. Along with these researches, there exist many cost effective hardware design which supports the digital signal processing (DSP) in framing of the occupancy sensing system [7]. The implementation of these sensors is constructed based on the cardio pulmonary action $[8,9]$. The limitation of the conventional occupancy sensor is that it uses highly non-invasive mechanism 
considered for the line of sight objects sensing and are used in the diagnosis of clinical applications. This manuscript aims to introduce cost effective machine learning mechanism for enhancing the accuracy of occupansy sensing using Doppler radar. The manuscript is organized with existing researches on detection schemes meant with power transmission lines, highlighted the research problem considered for the design and the solution introduced for the problem is briefed in section 1. The system implementation of the proposed system is described in section 2 through implementation strategy and algorithm implementation in section 3. The conclusive points of the manuscript is discussed in section 4 .

The various techniques which are associated with the occupancy sensing and the research trend is highlighted the work of Mane and Rao [10]. In Amayri et al. [11] have introduced a Bayesian network based model which helps to estimate the sensing occupancy. The furter investigation on occupancy sensing performance is described in Eltabie et al. [12] over the condition of under used spectrum. Carrying the spectrum usage in the occupancy sensing, the work of Hlophe et al. [13] have discussed the deep learning based stochastic approach. The incorporation of Internet of Things (IoT) with occupancy sensing is found in Yang et al. [14] where channel state information is considered. The machine learning approach with the motion sensing mechanism is introduced for the occupancy sensing in Wu et al. [15]. In the work of Cao et al. [16], the smart sension approach is presented which adapts the significant building mechanism with better power management. The humans counting based on the bio-signals through occupancy sensing is described in the work of Santra et al. [17]. The sensory data based object detection is provided in Luppe and Shabani [18]. The sensed data from the occupancy sensing is analysed by using the evidency theory in Nesa and Banerjee [19]. The work of Jin et al. [20] smart meter based occupancy sensing mechanism is presented to perform the power management. The bluethooth generated signals are used for the occupancy detection by Shen and Newsham [21]. The specific forms of sensors are used in the work of Liu et al. [22] for occupancy sensing by using the hidden markov model. The idea of environmental factor sharing is discussed in Jin et al. [23] for the occupancy sensing. The hybrid mechanism for enhancement of accuracy in detection process is presented in Khan et al. [24]. The spectrum sensing through the deep learning based stochastic approach is found in Liu et al. [25] and spectrum sension in Mariani et al. [26] incorporating the cognitive radio. The work of Albesa and Gasulla [27] has used the inductive links for the occupancy factor detection by adapting the idea of magnetic coupling. A capacitive modelling is performed in the work of Cooley et al. [28] and the work of Hossain [29] adapted the Posteriori mechanism. The inductuce principle incorporated in the work of George et al. [30] while disputes of text cluster in the work of Nikharth and Subrahmanyam [31] for occupancy sensing. The problems identified in the existing researches are higlighted below.

The review of literature clearly indicates that, the existing literature lacks significant work realted to the evalution of identification of the occupancy sensors and many of the approaches includes the use of the Dopper Radar based computation using wave signal interpretations using the intrinsic features realted to the physiological attributes. One of the critical requirement in the applications based on the occupancy require an accurate calssifcations of the actions of the occupants, whereas the traditional approaches lacks the capacity to classify the occupants actions or the typical activities in more computationaly effcienct and accurate way. The challenges lies in order to develop such classification models are its dependencies on the higher dimentions of the feature set from a large set of explicit sensors so that system model design becomes capable of handling either single class of ectivity dection or the multiple classes of the action or activity detection in more computational effciuent manner to minize the computational cost. Hence, the design approach of such classification models requires handling the huge veracity of the occupant's actions and activities in the region of occupant applications of interest. Therefore, the initial objective is to handle the issue is design a computational cost effective learning model which classifies the occupant actions categorically then the aim is to optimize the model with the indentifcation of more significant feature classes that impact well on the actions sub-spce classification in case of ambiguity so that the sepecifity and ensitivity of the models are acceptable.

The proposed system is designed and developed for improving the prior model [32] where the prime focus is offered towards constructing a robust mathematical framework in order to optimize the performance of radar-based sensing. The study introduces a novel form of design of the Doppler radar different from the traditional model in order to carry out an effective event classification of the subjects. The proposed architectural scheme for this sensing operation is shown in Figure 1.

The complete design of the proposed system is carried out using analytical modeling that perform developing certain attributes for assisting in classifying different forms of sensed event in order to facilitate precise sensing of subject occupancy. The proposed study also formulates a novel extraction of essential features in order to improvise the performance of classification on the basis of signals extracted from Doppler 
radar. The study mainly tracks down the mobility-based movement as the prime source of event generation where Doppler radar is used traking with respect to track points. The system carries out identification of the subjects on the basis of this track points. The system implementation begins with the investigation over the computation of such attibutes in order to plan out effective logical condition for incorporating accuracy while performing estimation operation. For this purpoe, the study frames up an algorithm that is responsible for choosing an elite form of an attribute in order to make the system independent from processing higher number of system attributes. This has a direct influence towards reducing computational complexity. The study will also have an inclusion of multiple variants of identification attributes using Doppler radar while it can perform different angular orientation for tracking the different events with defined and unknown orientation. The consecutive part of the section highlights about the implementation approach adopted in the proposed study.

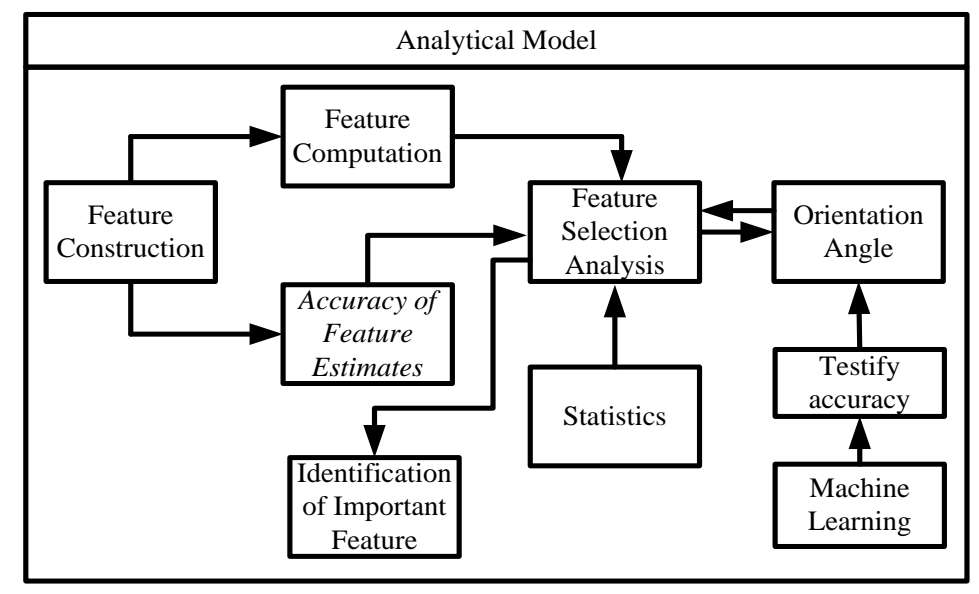

Figure 1. Proposed architecture of sensing and classification

\section{PROPOSED IMPLEMENTATION APPROACH}

This section discusses about the implementation approach towards frameing up a model that can enhance the performance of sensing of proposed system using its optimization approach. The proposed system targets to improve the performance of the system as a mechanism to carry out optimization without any form of dependencies of sophisticated resources. The discussion of the proposed system implementation is carried out with respect to the strategy adopted for the implementation, core design of system, and flow of execution for illustrating the algorithm steps.

\subsection{Strategy of implementation}

The important strategy of the implementation is about carrying out the optimization process for extraction of feature where the signals associated with the movement is collected by the Doppler radar. It should be noted that the sensing is carried out over different set of events. The proposed system make use of the static mechanism for performing feature extraction in presence of multiple number of fluctuation of environment variables e.g. time for settling, angle of orientation, quality of signal. Therefore, the study is more emphasized over information extraction in precise manner that is connected with the attributes for event. This operation positively influences the classification mechanism and computational performance of proposed system using machine learning. The next section illustatrates about core design of system.

\subsection{Core design of system}

The proposed study is initiated on the basis of the consideration that a Doppler radar is generating a specific form of signal in the form of modulated frequency of linearized form. The proposed studies consider the concept of event as set of specific events or actions created by the human movement as the prime indicator of the occupancy sensing. However, it is quite a complex task in order to understand the orientation of the actions exhibited by the human as they are highly unpredictable. However, there are always some set of actions that signifies tentative events. The Doppler radar collects the trajectory that is reflected from the parts of the body in the form of the modulated signals. At present, there are two discrete 
techniques in order to be used in occupancy sensing where one of the approaches is connected with kinematic system while another approach is associated with the motion-based attributes. In the existing approaches, majority of the usage is associated with the conventional kinematic modeling approach. Such model is completely designed on the basis of the investigation towards specific movement of the human body consisting of joint and points movement connected with the human body. As per the conventional system, it is believed that the Doppler signal for the human is accumulated from the multiple different parts of the target point of the human body that is believed to be characterized by trajectory with time varying traits as per the kinematic model. However, this model is restricted only to the scenario when the subject is found mobile over their feet. The devices associated with the motion capture are connected with further accuracy in the context of the extracted spectrogram of a human. While comparing with the kinematic modeling (that is found to have more dependencies on the computation of the temporal factor assoiated with the human body), such approach on the basis of the motion. This approach is capable of transforming the movement radar data collected from certain sensors in the form of the simulated data. This simulated version of the real signals uses the standard radar concept in order to compute the extracted signal. It will mean that the generated data is absolutely not in the generic form and it refers to certain explicit form of signal and hence the proposed system considers the data to be generated on the basis of motion controls.

From the conventional theory of Doppler radar system, it is believed that aggregation of interference and reponse of the target results in cumulative return signal that is extracted by the Doppler radar. The proposed study assumes that it implements suppression of clutter and therefore, the model performs representation of the remnant interference in the form of normal distributive noise. Therefore, the signal that is obtained from the source target point can be basically considered as the replicated copy of the shifted frequency characterized by delayed time associated with the forwarded signal. The next part of study implementation is more focused on extracting the features for better accuracy of performing occupant action sensing. At present, there are various forms of information that can be obtained from the event feed in the form of different actions. Hence, an explicit approach is presented in proposed system where the system considers three types of data i.e. training data, testing data, and features data. All these data acts as an input to the proposed system. The proposed study constructs the data structured of potential features which is formed using both test data and trained data. The data base actually represents certain specific set of movement of subjects and therefore the proposed system obtains the information associated with the identity of the subjects in both train and test data. All the obtained data is now normalized to obtain the maximum value. The proposed study performs an assessment to check if there is a purest form of the data with respect to the individual identity of the actions and subject. Finally, the proposed study obtains the normalized data with respect to the actions corresponding to the events.

The next part of the implementation is associated with the implication of the machine learning. The core motive of this part of the implementation is to testify usage of three different mechanism of machine learning approach i.e. deep learning approach, K-nearest neighboring algorithm (KNN), and Support Vector Machine. For this purpose, all the explicits features are loaded and statistical information is extracted. The proposed study extracts standard deviation from the features and checks if the obtained features (or standard deviation) are found more than ideal value of statistics. The study obtains all the samples with respect to the statistical feature which is further randomized. The next part of the implementation is about performing training of the data considering presence of error. The study selects the cumulative number of training data as well as considers the arbitrary flag of the trained data. This operation is followed by extracting the input of the training followed by extracting the corresponding target of the training. Owing to the adoption of layer-based machine learning system, the proposed study considers two equivalent layers and specific value of the input side with discrete definition of the number of classes. The primary operation of the proposed machine learning system initiates with the declaration of the appropriate attributes where the input size corresponds with the size of the trained data. This operation is also followed by initializing the anticipated mean activation function for the hidden units, weight reduction attribute and penalty attribute. The second step of operation of the proposed system is meant for performing the encoding mechanism. The implemention is then focused on performing optimization using standards of quasi-Newton mechanism which interestingly offers a better form of memory optimization. The presented optimization approach performs a computation of the hessian matrix in order to enhance the search space. This mechanism of deep learning approach offers better performance applicable over different domains inclusive of language, speech, vision, etc. Another significant advantageous feature of using deep neural network is the minimization of the dependencies for any sort of expensive engineering process that is considered to save maximum production time while practicing machine learning approach. At the same time, the adaptability charecteristic

Int J Elec \& Comp Eng, Vol. 10, No. 4, August 2020: 4093 - 4100 
of the deep learning approach is another suitable feature that increases the applicability of the machine learning approach. In simpler sense, it will mean that over an increasing set of the data, deep learning approach claims to offer enhanced performance in comparison to existing approaches.

The machine learning approach is to perform implications of the KNN algorithm that uses class membership as the representation of the output. The classification of an object is carried out with an aid of multiple selections of its neighboring nodes where the class is assigned an object with highest common among the specific integer number of nearest neighborhood. One interesting fact about the KNN algorithm is that it performs the approximation of the function locally and the system defers all the computation until and unless the classification is not carried out. Some of the essential charecteristics for which the KNN algorithm has been selected for performing training and testing are as follows: This algorithm is quite simple and highly spontaneous where the simplicity of the computation is carried out by reading all the values in the dataset. There is no specific assumption connected to the usage of the KNN algorithm unlike various existing linear regression algorithm as well as there is no inclusion of steps of the training. There is no model building process in KNN but only the indexing of the novel entries of data is carried out in it. Another significant charecteristics of KNN is that it spontaneously evolves. Capability to solve the multi-class problem is another interesting charecteristics of the KNN algorithm with enhance capability to adopt different distance models in it viz. i) Minkowski distance, ii) Manhattan distance, iii) Hamming distance, iv) Euclidean distance. The next part of the implementation for the machine learning will be Support Vector Machine (SVM). Basically, SVM is a type of the supervised learning model that is associated with enhanced classification performance. Along with performing linear classification operation, the SVM approach is capable of performing non-linear classification operation that is also termed as kernel-based operation. The implication of the SVM approach of the machine learning performs applying a hyperplane over a high dimensional space that is normally utilized for performing regression or classification operation. The conventional usage of SVM is reportedly found to be used in classification of signal, categorization of hypertext or text, recognization of handwritten characters. The prime reasons for wide range of availability are that-It is the most suitable for the application where the input data is very vague or does not have much clarity. Its applicability is quite high, especially in case if the data is semi-sructured or unstructured, which is the majority of the cases in present times.

\subsection{Execution flow}

The algorithm of the proosed system performs optimization of the sensing performance of the Doppler radar towards tracking down the dynamic events. Different record of an event is considered as an input for the proposed system that is particular to the actions of human. The study offers an outcome as feature that is extracted from the events. The algorithm carry out non-recursive operation for extraction features from the given scenatio of an event and extract the final feature. The steps of the algorithm are as follows:

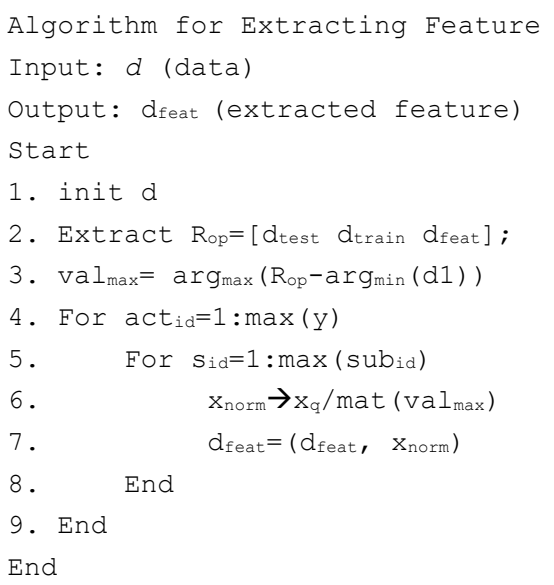

The elaborations of the essential algorithmic steps are: The first line of action of the proposed algorithm is to consider an event data $d$ as its input. Using this input value, the system obtains various forms of features in the form of different discrete action that are stored in $\mathrm{R}_{\mathrm{op}}$ matrix. This matrix has inclusion of $d_{\text {train }}$ trained data, $d_{\text {test }}$ test data, $d_{\text {feat }}$ data with feature. This operation is followed by obtaining val $\operatorname{lax}_{\max }$ highest possible value obtained from the sensed data of an event which is supported by dual attributes. The primary attribute is $R_{o p}$ while the secondary attribute will be $d_{1}$ that are essentially a matrix to store trained data and test data. The possibility of the highest value from the $\mathrm{d}_{1}$ matrix is $v_{\max }$ while the consecutive 
implementation of the algorithm takes this highest value with respect to $\mathrm{y}$ parameter that is essentially a matrix with all training and test data. The study takes the identity of the subject in order to frameup $\mathrm{x}_{\text {norm }}$ normalized matrix which two supporting attributes i.e. $\mathrm{x}_{\mathrm{q}}$ and $\operatorname{mat}\left(\mathrm{val}_{\max }\right)$. The matrix upon subjected to resizing operation is stored back in $x_{q}$ matrix that has possession of all the respective $d_{1}$ data associated with the specific event of action as well as its linked identity. All cumulative features are computed with an aid of the obtained $x_{n o r m}$ vector. The study also constructs $d_{\text {feat }}$ another matrix that stores values of cumulative actions linked with all the feature in order to represent a particular case of an occurred event. Machine learning approach is applied in next step which begins with selecting training parameters e.g. number of layers, decay attribute weight, sparsity weight, etc. An unsupervised learning algorithm is designed for reducing the dimension of the data in proposed machine learning approach. The study performs optimization operation using Broyden-Fletcher-Goldfarb-Shanno method in order to have a significant control over cost. The study applies feedforward neural network in order to perform training rounds using unsupervised methodology. The classification is carried out next on depending upon the attrbutes that are obtained from the learning method. The assessment of the study was carried out using other related training mechanism as exhibited in Figure 2.

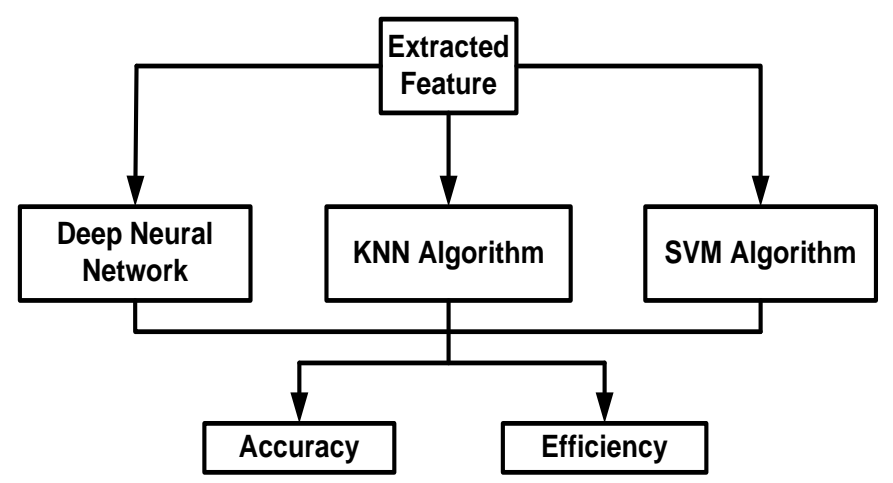

Figure 2. Feature extraction and training process

\section{RESULT ANALYSIS}

The outcomes of the proposed model are analyzed by standard dataset [33] that exhibits different activities captured for research which includes 10,299 instances of data sets and 561 attributes. The data dataset is characterized with multi-variate and time series analysis. The peroformance analysis of the system is done with comparision of recent machine learning approaches considering the scale of error rate and efficiency. The following Figures 3 and 4 gives the comparative analysis of error rate and efficiency with different techniques.

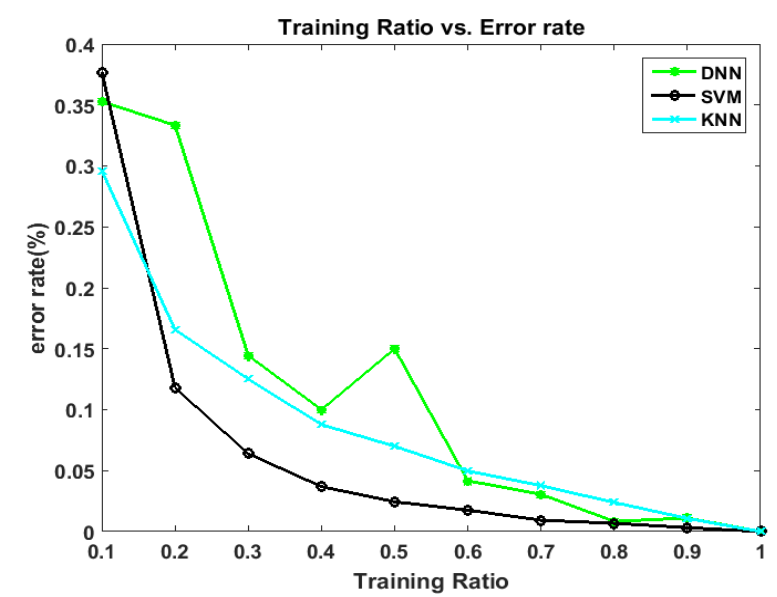

Figure 3 Study of Error Rate

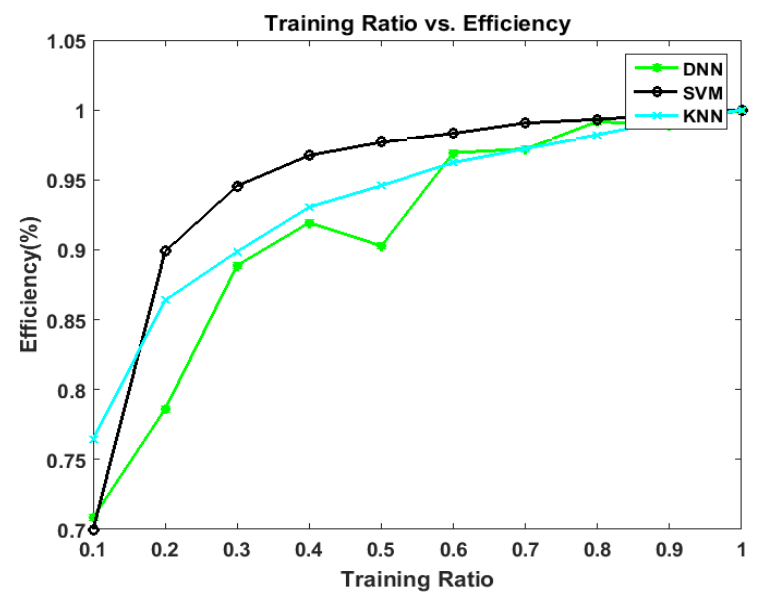

Figure 4. Comparative analysis of efficiency 
The study outcome shows that proposed system offers better efficiency with SVM and next better performance observed is for KNN while the efficiency of DNN is after that. The prime reason behind this outcome is-SVM offers better feature for L2 regularization that successfully resists from various over-fitting problems and hence accuracy factor of SVM is found to be higher than existing KNN as well as DNN as shown in Figure 4. At the same time, SVM also offers better stability performance even compared to KNN and DNN resulting in better efficiency even in increasing training ratio. Figure 4 exhibits that SVM outperforms KNN and DNN with the increased training ratio when both efficiency $(\%)$ and error rate $(\%)$ are concerned.

The investigation result shows that proposed framework offers better effectiveness with SVM. The performance analysis is observed for KNN and DNN efficiency later is after that. It is observed that SVM offers better features for L2 regularization that effectively opposes from different over-fitting issues and henceforth exactness factor of SVM is seen as higher than existing KNN just as DNN see Figure 4. Simultaneously, SVM likewise offers better stability which is analyzed with KNN and DNN leading to significant efficiency as well as improved training ration. Figure 4 displays that SVM beats KNN and DNN with the expanded preparing proportion when both error rate (\%) and effectiveness (\%) are concerned.

\section{CONCLUSION}

As the acooupancy detection is not limited only to the find the number of accoupants, where most popular models of computation like Doppler methods are used as an invasive approach, whereas the identification of the occupants' actions plays an imporyant role to build some context oriented applications. The dataset includes the sensor attributes weables of the devices of the users as an non-invasive approach to bring intrinsic interpreseations as oppose to the bio-signals taken from the invasive sesnors and applying computaionaly complex algorithm. The proposed learning models take the statistical coraltion as a feature vector in order to design the analytical framework. The framework includes evaluation of the different learning modles to validate its behavior in terms of the accuracy, time complexities and computational complexities. The approach of the framework design provides a simple and highly non-recrusive approach of the feature descriptors and their corelatuon as compared to the traditional approaches of the occupant classification. The model improvised a layers approach of convolutional neural network (LA-CNN) by means of optimization and its benchmarking with repects to the SVM as well as KNN outperforms its accuracy.

\section{REFERENCES}

[1] Yoganandini, A. P., and G. S. Anitha, "A cost effective computational design of maximum power point tracking for photo-voltaic cell," International Journal of Electrical and Computer Engineering (IJECE), vol. 9, no. 2, pp. 851-860, 2019.

[2] Angadi, Parvathi, M. Nagendra, and M. Hanumanthappa, "A novel framework for efficient identification of brain cancer region from brain MRI," International Journal of Electrical and Computer Engineering (IJECE), vol. 9, no. 2, pp. 1410-1417, 2019.

[3] Dipoala, W. S, "Microwave intrusion detector with threshold adjustment in response to periodic signals," U.S. Patent 5,581,237, no. 3, 1996.

[4] Weng, T, and Y. Agarwal, "From buildings to smart buildings-sensing and actuation to improve energy efficiency," IEEE Design \& Test of Computers, vol. 29, no. 4, pp. 36-44, 2012.

[5] Yavari, E., C. Song, V. Lubecke and O. Boric-Lubecke, "Is there anybody in there?: Intelligent radar occupancy sensors," IEEE Microwave Magazine, vol. 15, no. 2, pp. 57-64, 2014.

[6] Yavari, E., A. Lee, K. Pang, N. Andrew McCabe and O. Boric-Lubecke, "Radar and conventional occupancy sensors performance comparison," Asia-Pacific Microwave Conference, pp. 444-446, 2014.

[7] Sarkar, Abhijit, Mark Fairchild and Carl Salvaggio, "Integrated daylight harvesting and occupancy detection using digital imaging," Sensors, Cameras, and Systems for Industrial/Scientific Applications IX, vol. 6816, 2008.

[8] Yavari, E., C. Song, V. Lubecke, and O. Boric-Lubecke. "Is there anybody in there?: Intelligent radar occupancy sensors," IEEE Microwave Magazine, vol. 15, no. 2, pp. 57-64, 2014.

[9] Santra, A., R. Vagarappan Ulaganathan, and T. Finke, "Short-range millimetric-wave radar system for occupancy sensing application," IEEE sensors letters, vol. 2, no. 3, pp. 1-4, 2018.

[10] Mane, Preethi K., K. Narasimha Rao, "Granular mobility-factor analysis framework for enriching occupancy sensing with doppler radar," International Journal of Electrical and Computer Engineering (IJECE), vol. 8, no. 2, pp. 979-988, 2018.

[11] Amayri, Manar, Stephane Ploix, Hussain Kazmi, Quoc-Dung Ngo and E. L. Safadi, "Estimating occupancy from measurements and knowledge using the bayesian network for energy management," Journal of Sensors, vol. 2019, pp. 1-12, 2019.

[12] Eltabie, Omar M., Mohamed, F. Abdelkader, and A. M. Ghuniem, "Incorporating Primary occupancy patterns in compressive spectrum sensing," IEEE Access, vol. 7, pp. 29096-29106, 2019.

[13] Hlophe, Mduduzi C and Sunil BT Maharaj, "Spectrum occupancy reconstruction in distributed cognitive radio networks using deep learning," IEEE Access, vol. 7, pp. 14294-14307, 2019. 
[14] Yang, Jianfei, Han Zou, Hao Jiang, and Lihua Xie, "Device-free occupant activity sensing using wifi-enabled iot devices for smart homes," IEEE Internet of Things Journal, vol. 5, no. 5, pp. 3991-4002, 2018.

[15] Wu, Libo, Ya Wang, and Haili Liu, "Occupancy detection and localization by monitoring nonlinear energy flow of a shuttered passive infrared sensor," IEEE Sensors Journal, vol. 18, no. 21, pp. 8656-8666, 2018.

[16] Cao, Ningyuan, Justin T., Shreyas Sen, and Arijit R., "Smart sensing for HVAC control: Collaborative intelligence in optical and IR cameras," IEEE Transactions on Industrial Electronics, vol. 65, no. 12, pp. 9785-9794, 2018.

[17] Santra, A., R. V. Ulaganathan, and T. Finke, "Short-range millimetric-wave radar system for occupancy sensing application," IEEE sensors letters, vol. 2, no. 3, pp. 1-4, 2018.

[18] Luppe, Christopher and Amir Shabani, "Towards reliable intelligent occupancy detection for smart building applications," IEEE 30th Canadian Conference on Electrical and Computer Engineering, pp. 1-4, 2017.

[19] Nesa, Nashreen, and Indrajit Banerjee, "IoT-based sensor data fusion for occupancy sensing using dempster-Shafer evidence theory for smart buildings," IEEE Internet of Things Journal, vol. 4, no. 5, pp. 1563-1570, 2017.

[20] Jin, Ming, Ruoxi Jia and Costas J. Spanos, "Virtual occupancy sensing: Using smart meters to indicate your presence," IEEE Transactions on Mobile Computing, vol. 16, no. 11, pp. 3264-3277, 2017.

[21] Shen, Weiming and Guy Newsham, "Smart phone based occupancy detection in office buildings," IEEE 20th International Conference on Computer Supported Cooperative Work in Design, pp. 632-636, 2016.

[22] Liu, Pengcheng, Sing-Kiong Nguang and Ashton Partridge, "Occupancy inference using pyroelectric infrared sensors through hidden Markov model," IEEE Sensors Journal, vol. 16, no. 4, pp. 1062-1068, 2015.

[23] Jin, M, N. Bekiaris-Liberis, K. Weekly, C. J. Spanos and A. M. Bayen, "Occupancy detection via environmental sensing," IEEE Transactions on Automation Science and Engineering, vol. 15, no. 2, pp. 443-455, 2016.

[24] Khan, M. D Abdullah Al Hafiz, HM Sajjad Hossain and Nirmalya Roy, "Sensepresence: Infrastructure-less occupancy detection for opportunistic sensing applications," 16th IEEE International Conference on Mobile Data Management, vol. 2, pp. 56-61, 2015.

[25] Li, Bin, S. Li, A. Nallanathan, Y. Nan, C. Zhao and Z. Zhou, "Deep sensing for next-generation dynamic spectrum sharing: More than detecting the occupancy state of primary spectrum," IEEE Transactions on Communications, vol. 63, no. 7, pp. 2442-2457, 2015.

[26] Mariani, Andrea, S. Kandeepan, and A. Giorgetti, "Periodic spectrum sensing with non-continuous primary user transmissions," IEEE Transactions on Wireless Communications, vol. 14, no. 3, pp. 1636-1649, 2014.

[27] Albesa, J. and M. Gasulla, "Monitoring switch-type sensors via inductive coupling: Application to occupancy and belt detection in removable vehicle seats," IEEE Transactions on Power Electronics, vol. 27, no. 11 pp. 4472-4480, 2012.

[28] Cooley, John J., Al-Thaddeus Avestruz and Steven B. Leeb, "A retrofit capacitive sensing occupancy detector using fluorescent lamps," IEEE Transactions on Industrial Electronics, vol. 59, no. 4, pp. 1898-1911, 2011.

[29] Hossain, K. and B. Champagne, "Wideband spectrum sensing for cognitive radios with correlated subband occupancy," IEEE Signal Processing Letters, vol. 18, no. 1, pp. 35-38, 2010.

[30] George, B, H. Zangl, T. Bretterklieber and G. Brasseur, "A combined inductive-capacitive proximity sensor for seat occupancy detection," IEEE transactions on instrumentation and measurement, vol. 59, no. 5, pp. 1463-1470, 2010.

[31] Nikhath, A. Kousar and K. Subrahmanyam, "Feature selection, optimization and clustering strategies of text documents," International Journal of Electrical and Computer Engineering (IJECE), vol. 9, no. 2, pp. 1313-1320, 2019.

[32] Mane, Preethi K., and K. Narasimha Rao, "Review of Research progress, trends and gap in occupancy sensing for sophisticated sensory operation," Computer Science On-line Conference, pp. 212-222, 2018.

[33] "Human Activity Recognition Using Smartphones Data Set", https://archive.ics.uci.edu/ml/datasets/human+activity+recognition+using+smartphones, retrieved on 20-09-2019

\section{BIOGRAPHIES OF AUTHORS}

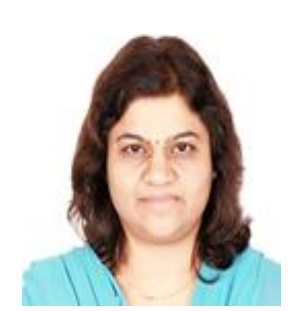

Preethi K Mane received her BE degree in Instrumentation Technology, in the year 1996 from Bangalore university and ME in Electronics from Bangalore university in the year 2000. She is pursuing $\mathrm{PhD}$ in Electronics and communication Engineering from Visvesvaraya Technological University. She has work experience of 21years in teaching and is currently serving as Associate Professor in the department of Electronics and Instrumentation Technology.

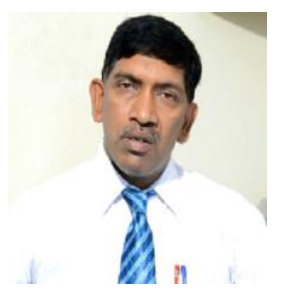

Dr K.Narasimha Rao has obtained his B.Tech, M.Tech and Ph D degrees in the year 1983, 1985 and 2011 respectively. He has 26 years of teaching experience. Presently, he is Professor Departmentt Of Instrumentation Technology, BMSCE, and Bangalore-19. His research interests are in the areas of energy efficient DC Drives, Digital Signal Processing, Process Control and Biomedical Signal Processing. 